\title{
Effect of a Lipid-Lowering Nutraceutical on Pulse-Wave-Velocity in Hy- percholesterolemic Patients with or without Chronic Kidney Disease
}

\author{
Arrigo F.G. Cicero ${ }^{1, *}$, Angelo Parini ${ }^{1}$, Martina Rosticci ${ }^{1}$, Barbara $^{1}$ Brancaleoni $^{1}$, Giuseppe Derosa ${ }^{2}$, \\ Elisa Grandi ${ }^{1}$ and Claudio Borghi ${ }^{1}$ \\ ${ }^{1}$ Medical and Surgical Sciences Dept, University of Bologna, Via Albertoni 15, 40138 Bologna, Italy \\ ${ }^{2}$ Internal Medicine and Therapeutics Dept., P.le Golgi, University of Pavia, Italy
}

\begin{abstract}
The recent literature has put a large interest on nutraceuticals with lipid lowering activity for the management of moderate cholesterolemia in subjects with mildly increased cardiovascular disease risk. The aim of our study was to evaluate the middle-term effect of a combined lipid-lowering nutraceutical on lipid parameters and aortic stiffness.

For this study we consecutively enrolled 40 moderately hypercholesterolemic outpatients with mild-to-moderate chronic kidney disease (CKD) and 40 cross-matched hypercholesterolemic subjects without CKD. All the patient were treated daily with a combined nutraceutical containing red yeast rice ( $3 \mathrm{mg}$ monacolin $\mathrm{K}$ ) and berberine (500 $\mathrm{mg})$. At the baseline and after 6 months of treatment we measured blood pressure, pulse wave velocity (PWV) and ematochemistry parameters.

No significant change has been observed during the study in both groups regarding body mass index, blood pressure, liver transaminases, creatinin-phosfokinase and eGFR.

In non CKD patients TC improved by $(-21,6 \%)$, LDL-Cholesterol by $(-24,2 \%)$, non HDL-Cholesterol $(-24,0 \%)$ and TG ($20,8 \%$ ).

In CKD patients TC improved by $(-21,1 \%)$, LDL-Cholesterol by $(-23,7 \%)$, non HDL-Cholesterol $(-23,9 \%)$ and TG ($20,4 \%)$.
\end{abstract}

No difference among groups has been observed regarding the effects on lipid metabolism.

The PWV has significantly improved in both groups $(\mathrm{p}<0.01)$ without differences between groups.

In summary, a combined lipid-lowering nutraceutical improved lipid pattern and PWV in both non CKD and mild-tomoderate CKD patients.

Keywords: Arterial stiffness, Berberine, Chronic Kidney Disease, Dietary supplements, Nutraceuticals, Pulse Wave Velocity, Red Yeast Rice.

\section{INTRODUCTION}

Chronic kidney disease (CKD) is an independent risk factor for cardiovascular disease [1]. There is clear evidence that pharmacologically treating hypercholesterolemia in moderateto-severe (pre-dialysis) CKD is associated to a significant improvement of cardiovascular disease risk [2], however there is less evidence supporting the pharmacological treatment of hypercholesterolemia in mild-to-moderate CKD.

The recent literature has put a large interest on nutraceuticals with lipid lowering activity for the management of moderate cholesterolemia in subjects with mildly increased cardiovascular disease risk [3]. However, a large number of these nutraceuticals are expensive, not widely available or need very high dosages assumed for long time and mostly not studied in CKD patients.

*Address correspondence to this author at the Medical and Surgical Sciences Department Via Albertoni 15, 40138 Bologna, Italy;

Tel: ++39 3498558017; Fax: ++39 0516826125;

E-mail: afgcicero@cardionet.it
Some lipid-lowering nutraceuticals have already shown to improve some marker of vascular function [4]. Pulse Wave Velocity (PWV), an instrumental marker of arterial stiffness, is an independent risk factor for cardiovascular disease both in general population [5] and in CKD subjects [6]. In CKD, PWV is associated to renal function decline and related mortality [7].

Both red yeast rice [8] and berberine [9] are lipidlowering nutraceuticals that have been shown to improve endothelial function in the middle-term, however their effect on PWV has not been clearly explored, especially in CKD patients.

In this context, the aim of our study was to evaluate the middle-term effect of a combined lipid-lowering nutraceutical on lipid parameters and aortic stiffness.

\section{METHODS}

For this study we consecutively enrolled 80 outpatients of the lipid clinic of the University hospital of Bologna. The enrolment criteria were: 
- Primary prevention for cardiovascular diseases.

- Estimated cardiovascular risk less than 5\% (SCORE risk) [10].

- LDL-cholesterolemia not at target after an adequate 3month period of life-style improvement, as for the latest European guidelines for dyslipidemia management [10].

- The half of the patients had to be affected by mild-tomoderate CKD, evaluated through the level of estimated glomerular filtration rate (eGFR) by the CKDEPI equation [11].

All patients were daily treated with a combined nutraceutical containing red yeast rice ( $3 \mathrm{mg}$ monacolin $\mathrm{K}$ ) and berberine $(500 \mathrm{mg})$ (kindly provided by Rottapharm-Madaus SpA, Monza, Italy), already tested to be safe in different patient populations, for instance elderly [12] and statinintolerant patients [13].

At baseline and after 6 months of treatment we measured blood pressure, pulse wave velocity (PWV) and hematochemistry parameters in order to evaluate the efficacy and safety in both non CKD and mild-to-moderate CKD patients.

The study was fully conducted in accordance with the Declaration of Helsinki. The study protocol was approved by the Ethical Committee of the University of Bologna and informed consent was obtained from all the patients before the inclusion in the study.

Blood pressure measurements were obtained from each patient (using the right arm) in the seated position, using a standard mercury sphygmomanometer (Erkameter 3000, ERKA, Bad Tolz, Germany; Korotkoff I and V) with a cuff of appropriate size. Blood pressure was measured by the same investigator at each visit, in the morning before daily drug intake and after the patient had rested for 10 minutes in a quiet room. Three successive blood pressure readings were obtained at 1-minute intervals, and the mean of the 3 readings was calculated. Blood pressure measurements were performed by physicians not belonging to the study in order to preserve study blindness by experimenters [14].

The pulse wave velocity (PWV) was calculated using the SphygmoCor System (Vx Pulse Wave Velocity, AtCorMedical Pty Ltd Head Office, West Ryde, Australia) with the patient in the supine position. The carotid and femoral pulse wave was analyzed, estimating the delay in the electrocardiogram (ECG) wave and calculating the PWV. The space measurements were taken with a measuring tape from the suprasternal notch to the carotid and femoral arteries at the sensor location $[15,16]$.

All plasma parameters were obtained after a 12 -h overnight fast. Venous blood samples were drawn by a nurse in all patients between 08.00 AM and 09.00 AM. Plasma used was obtained by addition of $\mathrm{Na}_{2}$-EDTA, $1 \mathrm{mg} / \mathrm{ml}$, and centrifuged at $3000 \mathrm{~g}$ for 15 minutes at $4^{\circ} \mathrm{C}$. Immediately after centrifugation, the plasma samples were frozen and stored at $-80^{\circ} \mathrm{C}$ for not more than 3 months. The following parameters were evaluated: total cholesterol (TC), HDL-cholesterol, triglycerides (TG), glucose, liver transaminases, and creatinine via standardized methods. All measurements were centrally performed in the laboratory of our department. Labora- tory technicians drew the blood samples, and a biologist performed the assays with standardized methods [17].

Compliance to the treatment was evaluated by a selfadministered questionnaire.

The statistical analysis of the data has been carried out with the help of the SPSS 21.0 statistical software (IBM Inc, Chicaco, IL, USA). The distribution normality of the tested parameters was evaluated by the Kolmogorov-Smirnov test. Continuous variables were compared by analysis of variance (ANOVA). Intervention effects were adjusted for additional potential confounders using analysis of covariance (ANCOVA). ANOVA was also used to assess the significance within and between groups. Outcome variables with a skewed distribution were transformed to a log scale before statistical testing. The statistical significance of the independent effects of CKD presence or less on the other variables was determined using ANCOVA. All the data are expressed as mean and standard deviation (SD). A "p" level less than 0.05 was considered significant for all tests.

\section{RESULTS}

Compliance to the treatment was overall good with no patient missing more than 3 tablets per month.

No significant difference has been observed regarding mean baseline values of the tested parameters between the two group of treatments ( $p>0.05$ for all), beyond eGFR that was significantly lower in CKD patients than in non-CKD ones (Table 1), as per protocol.

No adverse event was registered during the study. Subjective tolerability and acceptability of the treatment was quoted as good to optimal.

No significant change has been observed during the study in both groups regarding body mass index, blood pressure, liver transaminases, creatinin-phosphokinase and eGFR Table 2.

In non CKD, patients TC improved by $(-21,6 \%)$, LDLCholesterol by $(-24,2 \%)$, non HDL-Cholesterol $(-24,0 \%)$ and TG (-20,8\%).

In CKD patients, TC improved by $(-21,1 \%)$, LDLCholesterol by $(-23,7 \%)$, non HDL-Cholesterol $(-23,9 \%)$ and TG $(-20,4 \%)$.

No difference among groups has been observed regarding the effects on lipid metabolism.

The PWV has significantly improved in both groups without differences between groups (Fig. 1).

\section{DISCUSSION}

Increasing evidence supports the hypolipidaemic efficacy of some nutraceuticals [18]. The use of full dosed nutraceutical deserves however some tolerability concerns, because they could have statins- or metformin-like side effects [19]. However the use of combination of nutraceuticals with different but synergic mechanism of action at lower and more safe dosages appear to be an interesting alternative. Red yeast rice reduces cholesterolemia directly by inhibiting the liver 3-hydroxy-3-methyl-glutaryl-Coenzime A reductase, while berberine by inhibiting the cleavage of the receptor for LDL-cholesterol from the liver cell membranes [20]. 
Table 1. Baseline Characteristics of the Enrolled Patients

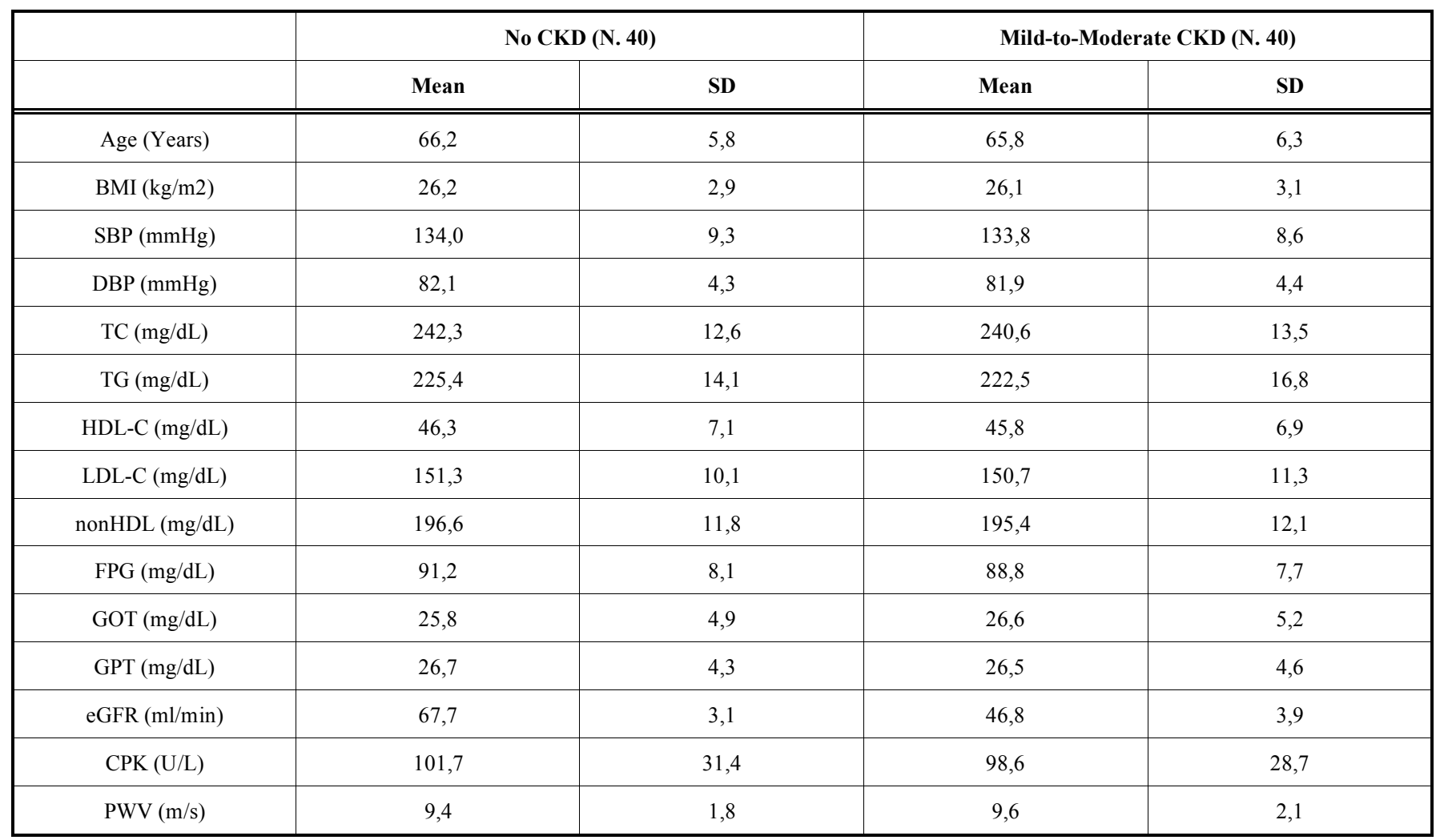

BMI= Body Mass Index, SBP= Systolic Blood Pressure, DBP= Diastolic Blood Pressure, TC= Total Cholesterol, TG= Triglycerides, HDL-C = High Density Lipoprotein Cholesterol, LDL-C $=$ Low Density Lipoprotein Cholesterol, FPG $=$ Fasting Plasma Glucose, GOT $=$ Glutamic Ossalacetate Transaminase, GPT $=$ Glutamic Pyruvic Transaminase, eGFR= estimated Glomerular Filtration Rate, CPK= Creatinine Phosfo Kinase, PWV= Pulse Wave Velocity

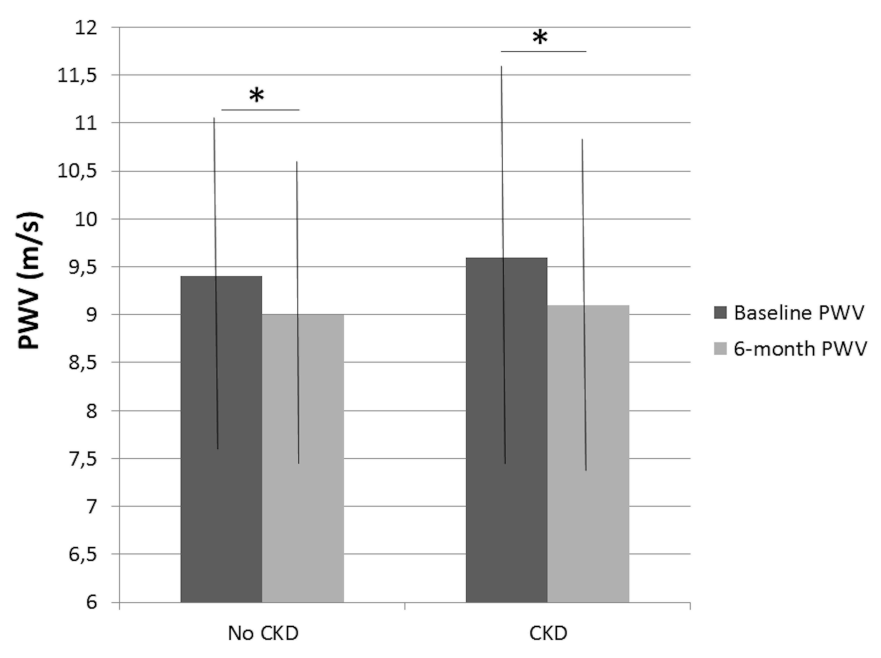

Fig. (1). Changes in pulse-wave velocity in subjects with and without mild-to-moderate chronic kidney disease after treatment with a lipidlowering nutraceutical

Table 2. Mean \pm SD Changes in the Studied Parameters

\begin{tabular}{|c|c|c|c|c|}
\hline & \multicolumn{2}{|c|}{ No CKD (N. 40) } & \multicolumn{2}{c|}{ Mild-to-Moderate CKD (N. 40) } \\
\hline & Mean & SD & Mean & SD \\
\hline \hline BMI (kg/m2) & $-0,3$ & 0,09 & $-0,2$ & 0,08 \\
\hline SBP (mmHg) & $-1,2$ & 2,4 & $-1,5$ & 2,3 \\
\hline
\end{tabular}


Table 2. contd...

\begin{tabular}{|c|c|c|c|c|}
\hline & \multicolumn{2}{|c|}{ No CKD (N. 40) } & \multicolumn{2}{|c|}{ Mild-to-Moderate CKD (N. 40) } \\
\hline DBP (mmHg) & 0,1 & 1,8 & 0,3 & 1,6 \\
\hline $\mathrm{TC}(\mathrm{mg} / \mathrm{dL})^{*}$ & $-52,4$ & 7,3 & $-50,8$ & 7,7 \\
\hline $\mathrm{TG}(\mathrm{mg} / \mathrm{dL}) *$ & $-46,8$ & 9,1 & $-45,5$ & 8,8 \\
\hline LDL-C (mg/dL)* & $-36,6$ & 6,2 & $-35,8$ & 6,2 \\
\hline nonHDL (mg/dL)* & $-47,2$ & 8,7 & $-46,7$ & 9,2 \\
\hline FPG (mg/dL) & $-3,3$ & 3,9 & $-2,9$ & 3,6 \\
\hline GOT (mg/dL) & $-2,4$ & 1,2 & $-2,5$ & 1,5 \\
\hline CPK (U/L) & 3,8 & 7,5 & 4,3 & 8,1 \\
\hline
\end{tabular}

$* \mathrm{p}<0.05$ versus baseline in both groups

$\mathrm{BMI}=$ Body Mass Index, $\mathrm{SBP}=$ Systolic Blood Pressure, $\mathrm{DBP}=$ Diastolic Blood Pressure, $\mathrm{TC}=$ Total Cholesterol, TG= Triglycerides, HDL-C $=$ High Density Lipoprotein Cholesterol, LDL-C $=$ Low Density Lipoprotein Cholesterol, FPG $=$ Fasting Plasma Glucose, GOT $=$ Glutamic Ossalacetate Transaminase, GPT $=$ Glutamic Pyruvic Transaminase, eGFR $=$ estimated Glomerular Filtration Rate, CPK= Creatinine Phosfo Kinase, PWV= Pulse Wave Velocity

Low-dosed red yeast rice and berberine assumed on top of a standard Mediterranean healthy diet, seems to be able to reduce LDL-C and TG by a mean $20 \%$ in different patient populations [21]. This effect has an obvious positive reflection on the metabolic syndrome management and on the patients' estimated cardiovascular risk [22].

In our study we also observed that the tested nutraceutical combination was able to reduce LDL-C and TG by respectively $24 \%$ and $21 \%$ in agreement with our previous results $[13,23]$ and with the results obtained by other independent researchers $[21,24]$. The innovative part of our results regards the confirmation of efficacy and safety of this approach in mild-to-moderate CKD patients, usually not enrolled in the previous trials. Moreover, we observed an improvement of PWV in both non CKD and CKD patients. The positive effect observed in healthy subjects is in agreement with what was already demonstrated in a short-term trial by Pirro et al. [25]. The combination of low-dosed red yeast rice and berberine has been proven to have some direct protective vascular effects, such as improvement of endothelial dysfunction, in another short term trial [26]. A further innovative aspect of our study is to have confirmed this vascular protective effect of the tested nutraceutical on a more long-term follow-up and, one more time, to have extended this observation to mild-to-moderate CKD patients.

Our study has some main limitations. The main one is that it is not a placebo-controlled double-blind clinical trial. However, the lipid-lowering activity of the tested nutraceuticals was already largely known from previous trials and the aim of our study was mainly concentrated on the tolerability and efficacy of low-dosed monascus and berberine in mildto-moderate CKD patients. Another defect of the trial was that we did not directly measured the GFR nor we measured other laboratory parameters potentially associated to PWV improvement as C-reactive protein or matrix metalloproteinases 2 and 9, that however we already demonstrated to be improved by the treatment with low-dosed red yeast rice and berberine [27]. The long-term effect of the tested treatment on patient's outcomes has to be evaluated with attention, in particular in patients with moderate CKD that have a significant increase in cardiovascular disease risk and that the guidelines suggest to be treated with statins [10]. However, the proposed approach could be considered for subjects with previous intolerance to statins and in subjects refusing statin treatment even after a correct explication of its need.

In conclusion, a combined lipid-lowering nutraceutical improved lipid pattern and PWV in both non CKD and mildto-moderate CKD patients.

\section{CONFLICT OF INTEREST}

The authors confirm that this article content has no conflicts of interest.

\section{ACKNOWLEDGEMENT} Italy

Institutional funding from the University of Bologna,
ABBREVIATION
$\mathrm{BMI}=$ Body Mass Index
$\mathrm{CPK}=$ Creatinine Phosfo Kinase
$\mathrm{DBP}=$ Diastolic Blood Pressure
eGFR $=$ estimated Glomerular Filtration Rate
FPG $=$ Fasting Plasma Glucose
GOT = Glutamic Ossalacetate Transaminase
GPT = Glutamic Pyruvic Transaminase 


$$
\begin{aligned}
& \text { HDL- }=\text { High Density Lipoprotein Cholesterol } \\
& \text { LDL- }=\text { Low Density Lipoprotein Cholesterol } \\
& \text { PWV }=\text { Pulse Wave Velocity } \\
& \text { SBP }=\text { Systolic Blood Pressure } \\
& \mathrm{TC}=\text { Total Cholesterol } \\
& \mathrm{TG}=\text { Triglycerides }
\end{aligned}
$$

\section{REFERENCES}

[1] Foster MC, Rawlings AM, Marrett E, et al. Cardiovascular risk factor burden, treatment, and control among adults with chronic kidney disease in the United States. Am Heart J 2013; 166(1): 1506.

[2] Heymann EP, Kassimatis TI, Goldsmith DJ. Dyslipidemia, statins, and CKD patients' outcomes - review of the evidence in the postsharp era. J Nephrol 2012; 25(4): 460-72.

[3] Nijjar PS, Burke FM, Bloesch A, Rader DJ. Role of dietary supplements in lowering low-density lipoprotein cholesterol: a review. J Clin Lipidol 2010; 4(4): 248-58.

[4] Cicero AF, Borghi C. Evidence of clinically relevant efficacy for dietary supplements and nutraceuticals. Curr Hypertens Rep 2013; 15(3): 260-7.

[5] Vlachopoulos C, Aznaouridis K, Stefanadis C. Prediction of cardiovascular events and all-cause mortality with arterial stiffness: a systematic review and meta-analysis. J Am Coll Cardiol 2010; 55(13): 1318-27.

[6] Rubin MF, Rosas SE, Chirinos JA, Townsend RR. Surrogate markers of cardiovascular disease in CKD: what's under the hood? Am J Kidney Dis 2011; 57(3): 488-97.

[7] Chen SC, Chang JM, Liu WC, http://www.ncbi.nlm.nih.gov/pubmed?term=Tsai

YC\%5BAuthor\%5D\&cauthor=true\&cauthor uid $=22855129$ et al. Brachial-ankle pulse wave velocity and rate of renal function decline and mortality in chronic kidney disease. Clin J Am Soc Nephrol 2011; 6(4): 724-32.

[8] Zhao SP, Liu L, Cheng YC, et al. Xuezhikang, an extract of cholestin, protects endothelial function through antiinflammatory and lipid-lowering mechanisms in patients with coronary heart disease. Circulation 2004; 110(8): 915-20.

[9] Cheng F, Wang Y, Li J, et al. Berberine improves endothelial function by reducing endothelial microparticles-mediated oxidative stress in humans. Int J Cardiol 2013; 167(3): 936-42.

[10] Task Force for the management of dyslipidaemias of the European Society of Cardiology (ESC) and the European Atherosclerosis Society (EAS), Catapano AL, Reiner Z, De Backer G, Graham I, Taskinen MR, Wiklund O, Agewall S, Alegria E, Chapman MJ, Durrington P, Erdine S, Halcox J, Hobbs R, Kjekshus J, Perrone Filardi P, Riccardi G, Storey RF, Wood D; ESC Committee for Practice Guidelines 2008-2010 and 2010-2012 Committees. ESC/EAS Guidelines for the management of dyslipidaemias: the Task Force for the management of dyslipidaemias of the European Society of Cardiology (ESC) and the European Atherosclerosis Society (EAS). Atherosclerosis 2011; 217 Suppl 1: S1-44.

[11] Matsushita K, Mahmoodi BK, Woodward M, et al. Chronic Kidney Disease Prognosis Consortium. Comparison of risk prediction using the CKD-EPI equation and the MDRD study equation for estimated glomerular filtration rate. JAMA 2012; 307(18): 1941-51.

[12] Marazzi G, Cacciotti L, Pelliccia F, et al. Long-term effects of nutraceuticals (berberine, red yeast rice, policosanol) in elderly hypercholesterolemic patients. Adv Ther 2011; 28(12): 1105-13.
[13] Cicero AFG, Derosa G, Bove M, Imola F, Borghi C, Gaddi AV. Long-term effectiveness and safety of a nutraceutical based approach to reduce cholesterolemia in statin intolerant subjects with and without metabolic syndrome. Curr Topics Nutrac Res 2009; 7(3-4): 121-6.

[14] Pickering TG, Hall JE, Appel LJ et al. Recommendations for blood pressure measurement in humans and experimental animals: part 1: blood pressure measurement in humans: a statement for professionals from the Subcommittee of Professional and Public Education of the American Heart Association Council on High Blood Pressure Research. Circulation 2005; 111(5): 697-716.

[15] Tomiyama H, Yamashina A. Non-invasive vascular function tests: their pathophysiological background and clinical application. Circ J 2010; 74(1): 24-33.

[16] Cicero AFG, Rosticci M, Gerocarni B, et al. Lactotripeptides effect on office and 24-hour ambulatory blood pressure, blood pressure stress answer, pulse wave velocity and cardiac output in patients with high-normal blood pressure or first degree hypertension: a randomized, double-blind, clinical trial. Hypertens Res 2011; 34(9): 1035-40.

[17] Cicero AFG, D'Addato S, Veronesi M, et al. Relationship between blood pressure, cholesterolemia and serum apolipoprotein B in a large population sample: the Brisighella Heart Study. J Hypertens 2012; 30(3): 492-6.

[18] Nijjar PS, Burke FM, Bloesch A, Rader DJ. Role of dietary supplements in lowering low-density lipoprotein cholesterol: a review. J Clin Lipidol 2010; 4(4): 248-58.

[19] Cicero AFG, Ferroni A, Ertek S. Tolerability and safety of commonly used dietary supplements and nutraceuticals with lipidlowering effects. Expert Opin Drug Saf 2012; 11(5): 753-66.

[20] Cicero AFG, Tartagni E, Borghi C. Nutraceuticals with lipidlowering activity: do they have any effect beyond cholesterol reduction? Clin Lipidol 2012; 7(5): 549-59.

[21] Trimarco B, Benvenuti C, Rozza F, Cimmino C.S., Giudice R, Crispo S. Clinical evidence of efficacy of red yeast rice and berberine in a large controlled study versus diet. Med J Nutr Metab 2011; 4(2): 133-40.

[22] Izzo R, de Simone G, Giudice R, et al. Effects of nutraceuticals on prevalence of metabolic syndrome and on calculated Framingham Risk Score in individuals with dyslipidemia. J Hypertens 2010; 28(7): 1482-7.

[23] Cicero AF, Rovati LC, Setnikar I. Eulipidemic effects of berberine administered alone or in combination with other natural cholesterol-lowering agents. A single-blind clinical investigation. Arzneimittelforschung 2007; 57(1): 26-30.

[24] Affuso F, Mercurio V, Ruvolo A, et al. A nutraceutical combination improves insulin sensitivity in patients with metabolic syndrome. World J Cardiol 2012; 4(3): 77-83.

[25] Pirro M, Lupattelli G, Del Giorno R, et al. Nutraceutical combination (red yeast rice, berberine and policosanols) improves aortic stiffness in low-moderate risk hypercholesterolemic patients. Pharma Nutr 2013; 1(2): 73-7.

[26] Affuso F, Ruvolo A, Micillo F, Saccà L, Fazio S. Effects of a nutraceutical combination (berberine, red yeast rice and policosanols) on lipid levels and endothelial function randomized, double-blind, placebo-controlled study. Nutr Metab Cardiovasc Dis 2010; 20(9): 656-61.

[27] Cicero AFG, De Sando V, Benedetto D, Cevenini M, Grandi E, Borghi C. Long-term efficacy and tolerability of a largely marketed multicomponent nutraceutical in overweight and normoweight dyslipidaemic patients. Nutrafoods 2012; 11(2): 15-21.

\footnotetext{
Received: August 27,2013 Revised: August 29, 2013
}

Accepted: August 29, 2013

(C) Cicero et al.; Licensee Bentham Open.

This is an open access article licensed under the terms of the Creative Commons Attribution Non-Commercial License (http://creativecommons.org/licenses/by-nc/3.0/) which permits unrestricted, non-commercial use, distribution and reproduction in any medium, provided the work is properly cited. 